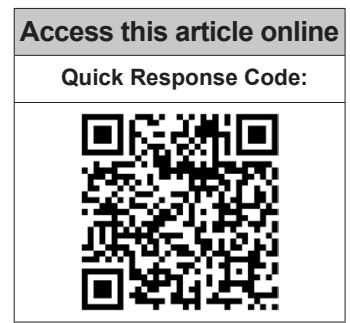

Website:

www.jponline.org

DOI:

10.4103/JLP.JLP_1_18
Department of Microbiology, Government Medical College, ${ }^{1}$ Department of Microbiology, SKIMS Medical College, Srinagar, Jammu and Kashmir, India

Address for correspondence: Dr. Asifa Nazir, 370 BBC, Shivpora, Srinagar - 190004 , Jammu and Kashmir, India.

E-mail: asifanazir@gmail. com

Submission: 01-01-2018 Accepted: 22-05-2018

\title{
Spectrum of candidal species isolated from neonates admitted in an Intensive Care Unit of teaching hospital of Kashmir, North India
}

\author{
Asifa Nazir, Talat Masoodi ${ }^{1}$
}

\begin{abstract}
:
BACKGROUND: Candidal infections are an important cause of morbidity and mortality in Neonatal Intensive Care Unit. Neonatal candidiasis is increasing in frequency, mainly because of increase in the survival of babies with low-birth weight, preterm births, advancement in medical field, life support systems, relative immunodeficiency, and extensive use of broad-spectrum antibiotics. Over the past few decades, there has been a progressive shift from the predominance of Candida albicans to nonalbicans Candida species.
\end{abstract}

AIMS AND OBJECTIVES: The objective of the current study was to know the prevalence of nonalbicans candidemia in neonates and their antifungal susceptibility pattern.

MATERIALS AND METHODS: In this study, a total of 424 samples from clinically diagnosed septicemic neonates were included. Identification of Candida isolates from these samples as well as their antifungal sensitivity testing was performed with Vitek 2 Compact (Biomerieux France) using Vitek 2 cards for identification of yeast and yeast-like organisms (ID-YST cards).

RESULTS: A total of $246 / 424(58.01 \%)$ cases were blood culture positive. Out of these, $80 / 246$ samples tested positive for candidemia (32.5\%). Candida tropicalis $(13.8 \%)$ was the predominant species isolated among the non-albicans Candida followed by Candida krusei (4.8\%), Candida parapsilosis (3.2\%), Candida guilliermondii (2.8\%), and Candida dubliniensis (2.0\%). We found an increase in the antifungal drug resistance, especially for the azole group of drugs, both in C. albicans and non-albicans Candida species. All the isolates were uniformly sensitive to micafungin, voriconazole, and caspofungin.

CONCLUSION: Candidemia in neonates is an ominous prognostic sign and is an important entity in our region. The present study highlights the mycological shift of Candida species in neonatal candidemia with a preponderance of nonalbicans Candida species.

Key words:

Antifungal susceptibility, Candida albicans, neonatal candidaemia, non-albicans candida

\section{Introduction}

The incidence and prevalence of 1 candidemia are on a rise in many countries worldwide. According to National Nosocomial Infection Surveillance, USA in the 1990s, Candida species remained the fourth most common bloodstream pathogen

This is an open access journal, and articles are distributed under the terms of the Creative Commons Attribution-NonCommercial-ShareAlike 4.0 License, which allows others to remix, tweak, and build upon the work non-commercially, as long as appropriate credit is given and the new creations are licensed under the identical terms.

For reprints contact: reprints@medknow.com accounting for $8 \%$ of all hospital-acquired bloodstream infections and is a cause of significant morbidity and mortality ${ }^{[1,2]}$ The Asian scenario regarding the incidence of candidemia is, however, not very clear due to a lack of multicentric studies. A 13-year long study on candidemia from a tertiary care hospital in Thailand showed a prevalence of $6.14 \%$ for Candida species among blood culture isolates. ${ }^{[3]}$ In India, there are a few studies indicating the increasing trend

How to cite this article: Nazir A, Masoodi T. Spectrum of candidal species isolated from neonates admitted in an Intensive Care Unit of teaching hospital of Kashmir, North India. J Lab Physicians 2018;10:255-9. 
of candidemia in some tertiary care hospitals. ${ }^{[4]}$ The mortality rate associated with candidemia worldwide is also high ranging from $10 \%$ to $49 \% .{ }^{[5]}$

Importance of Candida species in Neonatal Intensive Care Units (NICUs) is increasingly being recognized. Candida species accounts for $9 \%-13 \%$ of all blood isolates in NICUs. ${ }^{[6]} \mathrm{C}$. albicans is the most commonly isolated species and accounts for $50 \%-70 \%$ of cases of invasive candidiasis ${ }^{[2,7]}$ However, the recent studies suggest that, with the introduction of fluconazole and itraconazole, there is an increase in the prevalence of nonalbicans candidial septicemia. ${ }^{[8]}$ Candida tropicalis, Candida glabrata, and Candida parapsilosis are being increasingly isolated in patients of neonatal septicemia. ${ }^{[9]}$ Candida species can spread through vertical transmission from maternal flora or through horizontal transmission from hands of health-care workers. The risk factors associated with candidemia include the use of broad-spectrum antibiotics, low birth weight (LBW), prematurity, and intravenous catheter. ${ }^{[10]}$

Over the past year, we noticed an increase in the isolation rate of nonalbicans Candida species from cases of neonatal septicemia, which prompted us to undertake the present study; to analyze and evaluate the change in the species distribution of Candida species in neonatal septicemia and determine their in vitro antifungal susceptibility and the risk factors associated with their acquisition. Further speciation and susceptibility testing of Candida sp. is still not routinely being done at most of the centers and as such no reliable data are available from our region regarding the estimation of antifungal use in hospitals. Furthermore, because of considerable regional variability, the local epidemiological knowledge is critical in terms of prevention and management of invasive Candida infections and can guide to initiate empirical antibiotic treatment which is essential for the management of the neonatal sepsis.

\section{Materials and Methods}

This prospective observational study was conducted in the Department of Microbiology, Government Medical College, Srinagar, Kashmir, over a period of 1 year (January 2016 to December 2016). This study was approved by the Ethical Committees of our institute and informed consent was obtained from each patient's next of kin. Candidemia was defined as at least one positive blood culture for Candida species in the presence of signs and symptoms of sepsis.

With all aseptic precautions, about $1-2 \mathrm{ml}$ of blood is drawn from each neonate. Blood culture samples were incubated in BacTAlert 3D (Biomerieux, India ${ }^{\circledR}$ ) automated blood culture system. One milliliter of blood was inoculated into ready to use BacT/ALERT PF Plus culture bottles (yellow color coded) for pediatric use with all due precautions and shaken well. The culture bottles were loaded into the instrument after scanning the barcode of the bottle and incubated. Positive or negative culture bottles were determined by BacT/ALERT Microbial Detection System. Blood cultures were considered negative only after 7 days of incubation.

Positive samples were examined by microscopy of Gram-stained preparations and subcultured on blood agar plate, MacConkey agar plates, and Sabouraud dextrose agar slant with antibiotics but without cycloheximide (Hi-Media Pvt. Ltd., Mumbai, India) in aerobic atmosphere. The preliminary identification was done by colony morphology on SDA, chromogenic media (HiChrome, Himedia, Pvt., Ltd.), growth at $45^{\circ} \mathrm{C}$, germ tube test, and by carbohydrate fermentation and assimilation tests. ${ }^{[11]}$

Identification of the organism was confirmed with automated Vitek 2 compact 60 system (BioMerieux India ${ }^{\circledR}$ ) using Vitek 2 cards. The Vitek ID and AST cards were chosen according to the results of the Gram staining and used according to the manufacturer's instructions. The Vitek-2 ID and AST cards were logged and loaded into the Vitek-2 Compact system. Antifungal sensitivity was performed against amphotericin B, 5 flucytosine, fluconazole, caspofungin, voriconazole, and micafungin. The MICs obtained were resolved into the three clinical categories (susceptible, intermediate, and resistant), according to the interpretative criteria provided by the automated systems' recommendations (CLSI) guidelines (M100-S25) version 2015.

For the identified cases, we collected the demographic data, underlying disease, and the presence of risk factors.

Data were maintained in Microsoft Office Excel, and tests of proportions were used for analysis. Results were recorded in percentages.

\section{Results}

A total of 424 samples from clinically diagnosed septicemic neonates were received in the Department of Microbiology during the 1-year study period. A total of 246/424 (58.01\%) cases were blood culture positive. Out of these, $80 / 246$ samples tested positive for candidemia (32.5\%).

Among the positive samples for candidemia, non-albicans Candida were responsible for $82.85 \%$ cases, whereas $17.5 \%$ cases were due to Candida albicans. C. tropicalis (13.8\%) was the predominant species isolated among the nonalbicans Candida followed by Candida krusei (4.8\%), 
C. parapsilosis (3.2\%), Candida guilliermondii $(2.8 \%)$, and Candida dubliniensis (2.0\%) [Table 1].

Once we correlated our results of candidemia with the clinical presentation of the neonates and associated risk factors, it was observed in the present study that prematurity and LBW were the most common risk factors followed by prolonged antibiotic therapy, ventilator support, and prolonged central venous line [Table 2].

It was observed in our study that the most common clinical presentation was respiratory distress, followed by failure to thrive, lethargy, and convulsions [Table 3].

The majority of the $C$. albicans were resistant to commonly used antifungal drugs such as fluconazole (42\%),

Table 1: Distribution of microorganisms isolated from cases of neonatal septicemia $(n=246)$

\begin{tabular}{lc}
\hline & Number of isolates (\%) \\
\hline Candida species isolated $(n=80)$ & \\
Candida tropicalis & $34(13.8)$ \\
Candida albicans & $14(5.6)$ \\
Candida krusei & $12(4.8)$ \\
Candida parapsilosis & $8(3.2)$ \\
Candida guilliermondii & $7(2.8)$ \\
Candida dubliniensis & $5(2.0)$ \\
Gram-negative bacilli ( $n=96)$ & \\
Klebsiella spp. & $40(16.2)$ \\
Acinetobacter spp. & $28(11.3)$ \\
Escherichia coli & $14(5.6)$ \\
Enterobacter spp. & $8(4.8)$ \\
Others & $6(6.6)$ \\
Gram-positive bacilli ( $n=70)$ & \\
Coagulase-negative Staphylococcus & $41(16.6)$ \\
Staphylococcus aureus & $19(7.7)$ \\
Enterococcus spp. & $10(4.0)$ \\
\hline
\end{tabular}

Table 2: Risk factors associated with cases of neonatal candidemia $(n=80)$

\begin{tabular}{lc}
\hline Underlying clinical feature & $n(\%)$ \\
\hline Preterm & $63(78.5)$ \\
Low birth weight & $59(73.7)$ \\
Prolonged IV antibiotics & $58(72.5)$ \\
Ventilator support & $45(56.2)$ \\
Prolonged central venous line & $40(50)$ \\
\hline
\end{tabular}

$\mathrm{IV}=$ Intravenous

Table 3: Clinical presentations observed in cases of neonatal candidemia $(n=80)$

\begin{tabular}{lc}
\hline Sign/symptom & Number of cases \\
\hline Respiratory distress & $62(77.5)$ \\
Failure to thrive & $60(75)$ \\
Feed intolerance & $54(67.5)$ \\
Lethargy & $52(65)$ \\
Convulsions & $34(42.5)$ \\
Bleeding tendency & $21(26.2)$ \\
\hline
\end{tabular}

flucytosine (28\%), and amphotericin B (14\%). Among the non-albicans Candida sp., C. tropicalis showed $49 \%$ resistance to fluconazole, $42 \%$ resistance to flucytosine, and $10 \%$ resistance to amphotericin B. C. krusei and C. parapsilosis were more resistant to azoles, particularly fluconazole, than C. albicans. All the isolates were uniformly sensitive to micafungin, voriconazole, and caspofungin [Table 4].

\section{Discussion}

Sepsis remains the main cause of morbidity and mortality in NICUs, ${ }^{[12]}$ and candidemia is becoming a common occurrence in patients admitted in ICUs, especially during the past decade. ${ }^{[13]}$

In our study, isolation rate of Candida from neonatal septicemia cases was $18.86 \%$, which is similar to several other reports showing the frequency of isolation from $13.6 \%$ to $19.6 \%$ cases. $^{[10]}$ A notable feature of our study was the emergence of nonalbicans Candida (82.85\%) as a major cause of neonatal candidemia. Our findings are supported by other studies from different regions of India that have documented predominance of nonalbicans Candida over C. albicans in neonatal septicemia. ${ }^{[6,14]}$ A shift toward nonalbicans Candida was also noted by Kapila, Mendiratta et al., and Pfaller et al. ${ }^{[15-17]}$

The increased incidence of nonalbicans Candida is probably due to the greater use of invasive devices, broad-spectrum antibacterial agents, more extensive surgical procedures, and use of advance life support on various transplant patients and selection of less susceptible species by the pressure of antifungal agent such as fluconazole. ${ }^{[18]}$

In our study, C. tropicalis $(13.8 \%)$ was the most common species followed by C. albicans (5.6\%), Candida krusei $(4.8 \%)$, C. parapsilosis $(3.2 \%)$, C. guilliermondii $(2.8 \%)$, and C. dubliniensis (2.0\%).

Our study is comparable to the epidemiological studies carried out in different parts of our country indicating C. tropicalis as the common cause of nosocomial candidemia. ${ }^{[17,19,20]}$

C. tropicalis as a cause of fungemia in neonatal ICU have been linked to the presence of the fungus on the hands of the hospital personnel. The ability of this organism to produce clusters is one of its major virulence factors. Once introduced into the immunocompromised host, C. tropicalis may be more virulent than C. albicans and can rapidly progress from colonization to invasion.

A number of risk factors are responsible for the emergence of neonatal candidemia in the recent years. 
Table 4: Antifungal susceptibility profile of Candidal isolates

\begin{tabular}{lccccc}
\hline Candida species & \multicolumn{5}{c}{ Resistance (\%) to antifungals tested } \\
\cline { 2 - 6 } & Fluconazole, $\boldsymbol{n}(\%)$ & Flucytosine, $\boldsymbol{n}(\%)$ & Amphotericin B, $\boldsymbol{n}(\%)$ & Voriconazole, $\boldsymbol{n}(\%)$ & Capsofungin, $\boldsymbol{n}(\%)$ \\
\hline Candida tropicalis & $16(49)$ & $14(42)$ & $10(10)$ & 0 & 0 \\
Candida albicans & $5(42)$ & $4(28)$ & $2(14)$ & 0 & 0 \\
Issatchenkia orientalis & $6(50)$ & $4(35)$ & $2(16)$ & 0 & 0 \\
Candida parapsilosis & $1(12)$ & $1(12)$ & $1(12)$ & 0 & 0 \\
Meyerozyma guilliermondii & $1(14)$ & $1(14)$ & 0 & 0 & 0 \\
\hline
\end{tabular}

These include widespread use of broad-spectrum antibiotics, loss of mucosal immunity, colonization, LBW, and length of hospital stay. ${ }^{[21-23]}$ In our study, preterm birth $(78.5 \%)$ and LBW (73.5\%) were the most common associated findings present in neonates with candidemia. This is in concordance with the results of studies of other authors. ${ }^{[10,24-26]}$

It was observed in the present study that respiratory distress $(77.5 \%)$ was the most common clinical presentation, followed by failure to thrive $(60 \%)$, feed intolerance (54\%), and lethargy (57\%). Convulsion (34\%) and bleeding tendency (21\%) $(43.64 \%)$ were the less common clinical features seen in our cases. Similar findings have been reported by other authors also. ${ }^{[26]}$

In our study, we found an increase in the antifungal drug resistance, especially for the azole group of drugs, both in C. albicans and nonalbicans Candida species. Antifungal susceptibility testing revealed that most of the $C$. albicans species were resistant to commonly used antifungal drugs such as fluconazole ( $42 \%)$, flucytosine (28\%), and amphotericin B (14\%). Among the nonalbicans Candida sp., C. tropicalis showed $49 \%$ resistance to fluconazole, $42 \%$ resistance to flucytosine, and $10 \%$ resistance to amphotericin B.

Many authors have also noted increasing trends of fluconazole resistance as reported $37.5 \%$ by Gupta et al., ${ }^{[22]}$ $36 \%$ by Kothari and Sagar ${ }^{[27]}$ and $11.7 \%$ by Xess et al. ${ }^{[14]}$ C. krusei is innately resistant to fluconazole.

In other studies also, a variable range of resistance to flucytosine was reported ranging from $37 \%$ by Bhatt et al. ${ }^{[28]}$ to $0 \%$ by Pahwa et al. ${ }^{[29]}$

In the present study, resistance to amphotericin B among all Candida isolates ranged from $10 \%$ to $14 \%$. Similar results were reported by Bhatt et al. 8\%. ${ }^{[28]}$ Although amphotericin B has a rapid cidal action against most strains of Candida species (especially, C. albicans), it is not the first choice due to associated nephrotoxicity and the newer lipid formulation having a better cidal effect profile.

All the isolates were uniformly sensitive to micafungin, voriconazole, and caspofungin.

\section{Conclusion}

The present study stresses the clinical importance and mycological shift of Candida species in neonatal candidemia with a predominance of nonalbicans Candida species in our region. It is evident from the results of our study that routine identification of Candida isolates to the species level, and the detection of resistant strains by antifungal susceptibility test is essential. Furthermore, there is a continued need for surveillance of candidemia to monitor changes in the epidemiological features and antifungal susceptibility and also to develop and evaluate prevention strategies. Furthermore, speciation of Candida in neonatal candidemia and measures taken to reduce the risk factors wherever possible and can reduce morbidity, mortality, and antifungal drug pressure in the neonates.

\section{Financial support and sponsorship} Nil.

\section{Conflicts of interest}

There are no conflicts of interest.

\section{References}

1. Banerjee SN, Emori TG, Culver DH, Gaynes RP, Jarvis WR, Horan $\mathrm{T}$, et al. Secular trends in nosocomial primary bloodstream infections in the United States, 1980-1989. National Nosocomial Infections Surveillance System. Am J Med 1991;91:86S-9S.

2. Jarvis WR. Epidemiology of nosocomial fungal infections, with emphasis on Candida species. Clin Infect Dis 1995;20:1526-30.

3. Tritipwanit K, Chindamporn A, Suankratay C. Epidemiology of candidaemia at King Chulalongkorn Memorial Hospital, Thailand. J Infect Dis Antimicrob Agents 2005;22:59-69.

4. Sahni V, Agarwal SK, Singh NP, Anuradha S, SikdarS, Wadhwa A, et al. Candidemia - An under-recognized nosocomial infection in Indian hospitals. J Assoc Physicians India 2005;53:607-11.

5. Narang A, Agrawal PB, Chakrabarti A, Kumar P. Epidemiology of systemic candidiasis in a tertiary care neonatal unit. J Trop Pediatr 1998;44:104-8.

6. Baradkar VP, Mathur M, Kumar S, Rathi M. Candida glabrata emerging pathogen in neonatal sepsis. Ann Trop Med Public Health 2008;1:5-8.

7. Pfaller MA. Epidemiology of candidiasis. J Hosp Infect $1995 ; 30$ Suppl:329-38.

8. Chakrabarty A, Singh K, Das S. Changing face of nosocomial candidaemia. Indian J Med Microbiol 1999;17:160-6.

9. Wenzel RP. Nosocomial candidemia: Risk factors and attributable mortality. Clin Infect Dis 1995;20:1531-4. 
10. Agarwal J, Bansal S, Malik GK, Jain A. Trends in neonatal septicemia: Emergence of non-albicans Candida. Indian Pediatr 2004;41:712-5.

11. McGinnis MR. Yeast identification. In: Laboratory Handbook of Medical Mycology. New York: Academic Press; 1980. p. 337-73.

12. Carvalho PR, Feldens L, Seitz EE, Rocha TS, Soledade MA, Trotta EA, et al. Prevalence of systemic inflammatory syndromes at a tertiary pediatric Intensive Care Unit. J Pediatr (Rio J) 2005;81:143-8.

13. Baley JE, Kliegman RM, Fanaroff AA. Disseminated fungal infections in very low-birth-weight infants: Clinical manifestations and epidemiology. Pediatrics 1984;73:144-52.

14. Xess I, Jain N, Hasan F, Mandal P, Banerjee U. Epidemiology of candidemia in a tertiary care centre of North India: 5-year study. Infection 2007;35:256-9.

15. Kapila S. Indentification of Candida species in neonatal septicemia. Int J Contemp Pediatr 2016;3:601-5.

16. Mendiratta DK, Rawat V, Thamke D, Chaturvedi P, Chhabra S, Narang $\mathrm{P}$, et al. Candida colonization in preterm babies admitted to neonatal Intensive Care Unit in the rural setting. Indian J Med Microbiol 2006;24:263-7.

17. Pfaller MA, Diekema DJ, Rinaldi MG, Barnes R, Hu B, Veselov AV, et al. Results from the ARTEMIS DISK Global Antifungal Surveillance Study: A 6.5-year analysis of susceptibilities of Candida and other yeast species to fluconazole and voriconazole by standardized disk diffusion testing. J Clin Microbiol 2005;43:5848-59.

18. Saiman L, Ludington E, Pfaller M, Rangel-Frausto S, Wiblin RT, Dawson J, et al. Risk factors for candidemia in neonatal Intensive Care Unit patients. The National Epidemiology of Mycosis Survey Study Group. Pediatr Infect Dis J 2000;19:319-24.

19. Shivaprakasha S, Radhakrishnan K, Karim PM. Candida spp. Other than Candida albicans: A major cause of fungaemia in a tertiary care centre. Indian J Med Microbiol 2007;25:405-7.
20. Verma AK, Prasad KN, Singh M, Dixit AK, Ayyagari A. Candidaemia in patients of a tertiary health care hospital from North India. Indian J Med Res 2003;117:122-8.

21. Fraser VJ, Jones M, Dunkel J, Storfer S, Medoff G, Dunagan WC, et al. Candidemia in a tertiary care hospital: Epidemiology, risk factors, and predictors of mortality. Clin Infect Dis 1992;15:414-21.

22. Gupta N, Mittal N, Sood P, Kumar S, Kaur R, Mathur MD, et al. Candidemia in neonatal Intensive Care Unit. Indian J Pathol Microbiol 2001;44:45-8.

23. Singh K, Chakrabarti A, Narang A, Gopalan S. Yeast colonisation \& fungaemia in preterm neonates in a tertiary care centre. Indian J Med Res 1999;110:169-73.

24. Rani R, Mohapatra NP, Mehta G, Randhawa VS. Changing trends of Candida species in neonatal septicaemia in a tertiary North Indian hospital. Indian J Med Microbiol 2002;20:42-4.

25. Goel N, Ranjan PK, Aggarwal R, Chaudhary U, Sanjeev N. Emergence of non albicans Candida in neonatal septicemia and antifungal susceptibility: Experience from a tertiary care center. J Lab Physicians 2009;1:53-5.

26. Narain S, Shastri JS, Mathur M, Mehta PR. Neonatal systemic candidiasis in a tertiary care centre. Indian J Med Microbiol 2003;21:56-8.

27. Kothari A, Sagar V. Epidemiology of Candida bloodstream infections in a tertiary care institute in India. Indian J Med Microbiol 2009;27:171-2.

28. Bhatt M, Sarangi G, Paty BP, Mohapatra D, Chayani N, Mahapatra A, et al. Biofilm as a virulence marker in Candida species in nosocomial blood stream infection and its correlation with antifungal resistance. Indian J Med Microbiol 2015;33, Suppl S1:112-4

29. Pahwa N, Kumar R, Nirkhiwale S, Bandi A. Species distribution and drug susceptibility of Candida in clinical isolates from a tertiary care centre at Indore. Indian J Med Microbiol 2014;32:44-8. 